\title{
Ergodic Products and Powers on Compact Subsets of the $p$-adic Field \\ by
}

\author{
Nacima MEMIĆ
}

Presented by Feliks PRZYTYCKI

Summary. We study powers of ergodic functions on compact subsets of the $p$-adic field. The same question is considered for products of compact subsets of the $p$-adic field.

1. Introduction. H. Diao and C. E. Silva [6] studied rational functions on the $p$-adic field. They proved necessary conditions for rational functions to be locally isometric or measure preserving. They provided digraph representations for locally isometric invertible functions on compact subsets of the $p$-adic field. Anashin [1] gave a characterization of measure-preserving and ergodic 1-Lipschitz maps on the set $\mathbb{Z}_{p}$ of $p$-adic integers, which generalizes some of the results in [4], [5], 7].

In this work we analyse powers and products of ergodic functions on compact subsets of the $p$-adic field.

We begin with some basic definitions which can also be found in [6] and [8].

Let $X$ be a compact subset of the $p$-adic field $\mathbb{Q}_{p}$ and $f: X \rightarrow X$ an invertible function. The function $f$ is said to be locally isometric if there exists an integer $l$ such that

$$
|f(x)-f(y)|=|x-y| \quad \text { whenever } \quad|x-y| \leq p^{l} .
$$

It was proved in [6. Theorem 3.1] that any rational function $f$ satisfying these conditions is measure preserving and that $X$ consists of a union of $p^{-l} \mathbb{Z}_{p}$-cosets which form cycles or orbits of $f$. Following the same steps as

2010 Mathematics Subject Classification: Primary 11S82; Secondary 37P20, 11K41.

Key words and phrases: $p$-adic field, ergodic functions, transitive functions.

Received 8 July 2015; revised 25 June 2016.

Published online 25 August 2016. 
in the proof of [6, Theorem 3.1] it can be easily seen that this result can be extended to any invertible locally isometric function.

If the set $X$ can be written as $\biguplus_{i=1}^{n} B_{p^{l}}\left(y_{i}\right)$, where $l$ is as in $(*)$ and $B_{p^{k}}(z):=\left\{x \in \mathbb{Q}_{p}:|x-z| \leq p^{k}\right\}$, then for all $m \leq l, X$ can also be put in the form $X=\biguplus_{i=1}^{n p^{l-m}} B_{p^{m}}\left(x_{i}\right)$.

$f$ is said to be transitive modulo $p^{-m}$ if by permuting indices we can get $f\left(B_{p^{m}}\left(x_{i}\right)\right)=B_{p^{m}}\left(x_{i+1}\right)$ for $i \in\left\{1, \ldots, p^{l-m} n-1\right\}$ and $f\left(B_{p^{m}}\left(x_{p^{l-m}}\right)\right)=$ $B_{p^{m}}\left(x_{1}\right)$. In other words the $f$-orbit of $B_{p^{m}}\left(x_{1}\right)$ is the whole $X$.

It was proved in [2, Theorem 4.23] and [2, Proposition 4.35] that an invertible 1-Lipschitz function $f$ is ergodic on $\mathbb{Z}_{p}$ if it is transitive modulo $p^{-m}$ for all $m \leq l$. Following the steps in the proof of [ $[$, Theorem 3.2] which was formulated for rational functions, we can see that [2, Theorem 4.23] and [2, Proposition 4.35] are also valid on any compact subset of $\mathbb{Q}_{p}$.

\section{Main results}

Lemma 2.1. Let $X=\biguplus_{i=1}^{n} B_{p^{l}}\left(x_{i}\right)$ be a compact subset of $\mathbb{Q}_{p}$, where $l$ is an arbitrary integer. Let $f: X \rightarrow X$ be invertible and locally isometric such that $|f(x)-f(y)|=|x-y|$ whenever $|x-y| \leq p^{l}$. Let $k$ be any positive integer.

(i) If $k$ and $n$ are not relatively prime, then $f^{k}$ is not transitive modulo $p^{-l}$.

(ii) If $k$ and $n$ are relatively prime, then $f^{k}$ is transitive modulo $p^{-l}$ if and only if $f$ is transitive modulo $p^{-l}$.

Proof. It is clear that if $f$ is not transitive modulo $p^{-l}$ then no power of $f$ can be transitive. Therefore, it suffices to prove this result with the assumption that $f$ is transitive modulo $p^{-l}$. Without loss of generality we may assume that

$$
f\left(B_{p^{l}}\left(x_{i}\right)\right)=B_{p^{l}}\left(x_{i+1}\right) \text { for } i \in\{1, \ldots, n-1\} \text { and } f\left(B_{p^{l}}\left(x_{n}\right)\right)=B_{p^{l}}\left(x_{1}\right),
$$

so that

$$
f^{s-1}\left(B_{p^{l}}\left(x_{1}\right)\right)=B_{p^{l}}\left(x_{s(\bmod n)}\right), \quad \forall s \geq 2 .
$$

(i) Let $k=s d$ and $n=t d$, where $s$ and $t$ are relatively prime and $d>1$. Then

$$
\left(f^{k}\right)^{t}\left(B_{p^{l}}\left(x_{1}\right)\right)=\left(f^{n}\right)^{s}\left(B_{p^{l}}\left(x_{1}\right)\right)=B_{p^{l}}\left(x_{1}\right) .
$$

The result follows since $t<n$.

(ii) Suppose that $k$ and $n$ are relatively prime. If $f^{k}$ is not transitive then there exists $i \in\{2, \ldots, n\}$ such that $f^{k s}\left(B_{p^{l}}\left(x_{1}\right)\right) \neq B_{p^{l}}\left(x_{i(\bmod n)}\right)$ for every positive integer $s$. From $(2.1)$ we see that $i-1 \neq k s(\bmod n)$ or equivalently $i-1 \neq k s-r n$ for all nonnegative integers $r, s$, which obviously contradicts the Euclidean algorithm. 
THEOREM 2.2. Let $X=\biguplus_{i=1}^{n} B_{p^{l}}\left(x_{i}\right)$ be a compact subset of $\mathbb{Q}_{p}$, where $l$ is an arbitrary integer, and let $k$ be any positive integer. Let $f: X \rightarrow X$ be invertible and locally isometric such that $|f(x)-f(y)|=|x-y|$ whenever $|x-y| \leq p^{l}$. Then $f^{k}$ is ergodic if and only if:

(1) $f$ is ergodic,

(2) $p \cdot n$ and $k$ are relatively prime.

Proof. Suppose that (1) and (2) are true. By Lemma 2.1 we infer that $f^{k}$ is transitive modulo $p^{-s}$ for every integer $s \leq l$.

On the other hand, if (1) is not valid then obviously $f^{k}$ is not ergodic. Moreover, if $f$ is ergodic and (2) is not valid, Lemma 2.1 implies that $f^{k}$ is not transitive modulo $p^{l-1}$, so $f^{k}$ is not ergodic.

Proposition 2.3. Let $X=\biguplus_{i=1}^{n} B_{p^{l}}\left(x_{i}\right)$ be a compact subset of $\mathbb{Q}_{p}$, where $l$ is an arbitrary integer, and let $k$ be any positive integer. Let $f$ : $X \rightarrow X$ be invertible and locally isometric such that $|f(x)-f(y)|=|x-y|$ whenever $|x-y| \leq p^{l}$. Assume in addition that $f$ is transitive modulo $p^{-l}$. Then $f$ is ergodic if and only if for every subset $Y=\biguplus_{s=1}^{m} B_{p^{l}}\left(x_{i_{s}}\right)$ of $X$, the function $g: Y \rightarrow Y$ defined by

$$
g(x)= \begin{cases}f^{i_{s+1}-i_{s}}(x), & x \in B_{p^{l}}\left(x_{i_{s}}\right), s \in\{1, \ldots, m-1\}, \\ f^{i_{1}-i_{m}+n}(x), & x \in B_{p^{l}}\left(x_{i_{m}}\right)\end{cases}
$$

is ergodic.

Proof. It suffices to prove the claim for $Y$ of the form $\biguplus_{i=1}^{n-1} B_{p^{l}}\left(x_{i}\right)$ and show that the choice of $x_{n}$ is arbitrary and can be replaced by any other element from $\left\{x_{1}, \ldots, x_{n-1}\right\}$. Indeed, if the previous assertion is proved then recursively we can generalise the result. In this way we would also have proved that $f$ is ergodic if and only if there exists one subset $Y$ composed of a disjoint union of $p^{-l} \mathbb{Z}_{2}$-cosets on which $g$ is ergodic.

First suppose that $f$ is ergodic. Let $m<l$. For every $y \in B_{p^{l}}\left(x_{n-1}\right)$ we have $g\left(B_{p^{m}}(y)\right)=f^{2}\left(B_{p^{m}}(y)\right) \subset B_{p^{l}}\left(x_{1}\right)$. Thus the $g$-orbit of $B_{p^{m}}\left(x_{1}\right)$ is its $f$-orbit from which we have removed the set $B_{p^{l}}\left(x_{n}\right)$. Hence this is in fact the whole set $Y$ where each $p^{-m} \mathbb{Z}_{2}$-coset appears only once. By [6, Theorem 3.2 ] we conclude that $g$ is ergodic.

Suppose that for some $m<l, f$ is not transitive modulo $p^{-m}$. Then the $f$-orbit of $B_{p^{m}}(y)$ is strictly contained in $X$ for every $y \in B_{p^{l}}\left(x_{n-1}\right)$. Since $f$ is transitive modulo $p^{-l}$, the intersection of the $f$-orbit of $B_{p^{m}}(y)$ with any of the balls $B_{p^{l}}\left(x_{i}\right), i \in\{1, \ldots, n\}$, is not empty and it is strictly contained in that ball. Since $g(y)=f^{2}(y)$ is also in the $f$-orbit of $y$, we conclude that the $g$-orbit of $B_{p^{m}}(y)$ remains strictly contained in $Y$, hence $g$ cannot be transitive modulo $p^{-m}$. 
By means of the characterization of ergodic 1-Lipschitz functions on $\mathbb{Z}_{2}$ given in [9], 3] and Proposition 2.3 we suggest an ergodicity test for some subsets of $\mathbb{Z}_{2}$, for example a union of two $4 \mathbb{Z}_{2}$-cosets as shown in the example below.

First we recall Yurova and Anashin's theorem on ergodicity of 1-Lipschitz functions on $\mathbb{Z}_{2}$.

Theorem 2.4 (Yurova and Anashin [9], 3], 11). A 1-Lipschitz function $f$ is ergodic on $\mathbb{Z}_{2}$ if and only if the following conditions are satisfied:

(1) $f(0)=1(\bmod 2)$,

(2) $f(0)+f(1)=3(\bmod 4)$,

(3) $\frac{f(2)-f(0)+f(3)-f(1)}{2}=2(\bmod 4)$,

(4) $\left|f(m)-f\left(m-2^{n}\right)\right|=2^{-n}$ for all $m \in\left\{2^{n}, \ldots, 2^{n+1}-1\right\}$,

(5) $2^{-n+1} \sum_{m=2^{n-1}}^{2^{n}-1}\left(f(m)-f\left(m-2^{n-1}\right)\right)=0(\bmod 4)$ for all $n \geq 3$.

EXAMPle 2.5. Let $g: 4 \mathbb{Z}_{2} \cup 1+4 \mathbb{Z}_{2} \rightarrow 4 \mathbb{Z}_{2} \cup 1+4 \mathbb{Z}_{2}$ be isometric invertible and transitive modulo 4 . Then $g$ is ergodic if and only if:

- $\frac{g(1)-g(0)-1}{2}=2(\bmod 4)$,

- $\sum_{m=4,5}(g(m)-g(m-4))=2^{3}\left(\bmod 2^{4}\right)$,

- $\sum_{m=0,1(\bmod 4)}\left(g(m)-g\left(m-2^{n-1}\right)\right)=0\left(\bmod 2^{n+1}\right)$ for $n \geq 4$,

where the last sum over $m$ is taken over $m \in\left\{2^{n-1}, \ldots, 2^{n}-1\right\}$.

Proof. Let $f: \mathbb{Z}_{2} \rightarrow \mathbb{Z}_{2}$ be defined as follows:

$$
f(x)= \begin{cases}g(x), & x \in 4 \mathbb{Z}_{2}, \\ x+2, & x \in 1+4 \mathbb{Z}_{2}, \\ x-1, & x \in 3+4 \mathbb{Z}_{2}, \\ g(x-1), & x \in 2+4 \mathbb{Z}_{2} .\end{cases}
$$

Notice that $f$ and $g$ are equal on $4 \mathbb{Z}_{2}$ and $g=f^{3}$ on $1+4 \mathbb{Z}_{2}$. Then $f$ and $g$ are related as in Proposition 2.3, which implies that they are either simultaneously ergodic or non-ergodic. Conditions (1), (2) and (4) of Theorem 2.4 are obviously satisfied for $f$.

Now we analyse under what conditions $f$ satisfies condition (3) of Theorem 2.4. Since

$$
\frac{f(2)-f(0)+f(3)-f(1)}{2}=\frac{g(1)-g(0)-1}{2},
$$


$f$ satisfies condition (3) of Theorem 2.4 if and only if

$$
\frac{g(1)-g(0)-1}{2}=2(\bmod 4) \text {. }
$$

Similarly,

$$
\begin{aligned}
& \sum_{m=2^{n-1}}^{2^{n}-1}\left(f(m)-f\left(m-2^{n-1}\right)\right) \\
& =\left(\sum_{m=0(\bmod 4)}+\sum_{m=1(\bmod 4)}+\sum_{m=2(\bmod 4)}+\sum_{m=3(\bmod 4)}\right)\left(f(m)-f\left(m-2^{n-1}\right)\right) \\
& =\sum_{m=0(\bmod 4)}\left(g(m)-g\left(m-2^{n-1}\right)\right) \\
& +\sum_{m=2(\bmod 4)}\left(g(m-1)-g\left(m-2^{n-1}-1\right)\right)+\sum_{m=1,3(\bmod 4)} 2^{n-1} \\
& =\sum_{m=0,1(\bmod 4)}\left(g(m)-g\left(m-2^{n-1}\right)\right)+2^{n-1} 2^{n-2} .
\end{aligned}
$$

Therefore, $f$ satisfies condition (5) of Theorem 2.4 if and only if

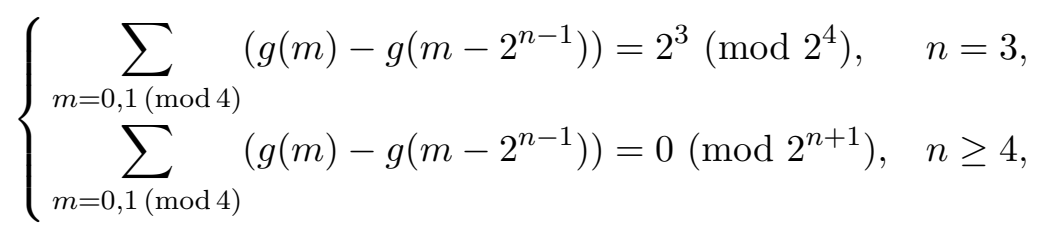

where the sums are taken over $m \in\left\{2^{n-1}, \ldots, 2^{n}-1\right\}$.

Theorem 2.6. Let $X^{j}=\biguplus_{i=1}^{n^{j}} B_{p^{l}}\left(x_{i}^{j}\right), j \in\{1, \ldots, m\}$, be compact subsets of $\mathbb{Q}_{p}$, where $l$ is an arbitrary integer. For every $j \in\{1, \ldots, m\}$ let $f_{j}: X^{j} \rightarrow X^{j}$ be invertible and locally isometric such that $\left|f_{j}(x)-f_{j}(y)\right|=$ $|x-y|$ whenever $x, y \in X^{j}$ with $|x-y| \leq p^{l}$. Let $k_{j}, j \in\{1, \ldots, m\}$, be any positive integers. Then $f_{1}^{k_{1}} \times \cdots \times f_{m}^{k_{m}}: X^{1} \times \cdots \times X^{m} \rightarrow X^{1} \times \cdots \times X^{m}$ is ergodic if and only if

(1) $f_{j}, j \in\{1, \ldots, m\}$, are ergodic,

(2) $\mathrm{pn}^{j}$ and $k_{j}$ are relatively prime for every $j \in\{1, \ldots, m\}$,

(3) $n^{i}$ and $n^{j}$ are relatively prime for all $i \neq j$ from $\{1, \ldots, m\}$.

Proof. We first handle the special case when $k_{j}=1$ for all $j \in\{1, \ldots, m\}$. We will prove that $f_{1} \times \cdots \times f_{m}$ is ergodic if and only if conditions (1) and (3) are satisfied.

It is easily seen that if some $f_{j}$ is not ergodic then $f_{1} \times \cdots \times f_{m}$ cannot be ergodic. Now assume that all $f_{j}$ are ergodic.

Without loss of generality we may assume that $n^{1}$ and $n^{2}$ are not relatively prime. Let $M$ be the smallest common multiple of $n^{1}, \ldots, n^{m}$. 
There exist integers $s$ and $t$ such that $M=s n^{1}=t n^{2}$. We will show that for each $n \in \mathbb{N}$,

$$
\left.\left(f_{1} \times \cdots \times f_{m}\right)^{n}\left(B_{p^{l}}\left(x_{1}^{1}\right) \times \cdots \times B_{p^{l}} x_{1}^{m}\right)\right)
$$

differs from

$$
B_{p^{l}}\left(x_{1}^{1}\right) \times B_{p^{l}}\left(f_{2}^{t n^{2}+1}\left(x_{1}^{2}\right)\right) \times B_{p^{l}}\left(t_{3}\right) \times \cdots \times B_{p^{l}}\left(t_{m}\right),
$$

for every $t_{j} \in X^{j}, j \geq 3$.

Indeed, suppose that for some positive integer $n$ we have

$$
B_{p^{l}}\left(x_{1}^{1}\right)=f_{1}^{n}\left(B_{p^{l}}\left(x_{1}^{1}\right)\right)
$$

and

$$
f_{2}^{t n^{2}+1}\left(B_{p^{l}}\left(x_{1}^{2}\right)\right)=f_{2}^{n}\left(B_{p^{l}}\left(x_{1}^{2}\right)\right) .
$$

If $f_{1}$ and $f_{2}$ are ergodic then $n$ must be a multiple of $n^{1}$ and $f_{2}^{t n^{2}}\left(B_{p^{l}}\left(x_{1}^{2}\right)\right)$ $=B_{p^{l}}\left(x_{1}^{2}\right)$. Hence, $f_{2}^{n-1}\left(B_{p^{l}}\left(x_{1}^{2}\right)\right)=B_{p^{l}}\left(x_{1}^{2}\right)$ and $n-1$ is a multiple of $n^{2}$. It follows that $n=r n^{1}=t^{\prime} n^{2}+1$, but this is impossible if $n^{1}$ and $n^{2}$ are not relatively prime.

Now, suppose that (1) and (3) are satisfied. For every $j \in\{1, \ldots, m\}$, let $i_{j} \in\left\{1, \ldots, n^{j}\right\}$ be arbitrary. We will show that

$$
B_{p^{l}}\left(x_{i_{1}}^{1}\right) \times \cdots \times B_{p^{l}}\left(x_{i_{m}}^{m}\right)=\left(f_{1} \times \cdots \times f_{m}\right)^{n}\left(B_{p^{l}}\left(x_{1}^{1}\right) \times \cdots \times B_{p^{l}}\left(x_{1}^{m}\right)\right)
$$

for some integer $n$.

Indeed, for each $j \in\{1, \ldots, m\}$ there exists an integer $r_{j}$ such that

$$
r_{1} n^{1}+i_{1}=r_{2} n^{2}+i_{2}=\cdots=r_{m} n^{m}+i_{m} .
$$

The result follows immediately by setting $n=r_{1} n^{1}+i_{1}$.

Now, let $k_{j}, j \in\{1, \ldots, m\}$, be any positive integers and assume that conditions (1)-(3) are satisfied. By Theorem 2.2, condition (2) implies that each $f_{j}^{k_{j}}, j \in\{1, \ldots, m\}$, is ergodic. Then the first part of the proof shows that $f_{1}^{k_{1}} \times \cdots \times f_{m}^{k_{m}}$ is ergodic.

Conversely, if $f_{1}^{k_{1}} \times \cdots \times f_{m}^{k_{m}}$ is ergodic then by the first part of the proof we see that condition (3) is satisfied and each $f_{j}^{k_{j}}, j \in\{1, \ldots, m\}$, is ergodic. Similarly, Theorem 2.2 implies that conditions (1) and (2) are satisfied.

REMARK 2.7. In [2, Theorem 4.51] it was proved that there is no ergodic uniformly differentiable function with integer-valued partial derivatives modulo $p$ on $\mathbb{Z}_{p}^{m}$ for $m \geq 2$. From condition (3) we can see that Theorem 2.6 is stated in a different context. Namely, the product is taken between mutually different sets $X_{j}, j \in\{1, \ldots, m\}$. Hence, Theorem 2.6 cannot be applied on $\mathbb{Z}_{p}^{m}$.

Acknowledgements. I would like to thank the referees for their remarks and suggestions. 


\title{
References
}

[1] V. Anashin, Ergodic transformations in the space of p-adic integers, in: p-Adic Mathematical Physics, AIP Conf. Proc. 826 (2006), 3-24.

[2] V. Anashin and A. Khrennikov, Applied Algebraic Dynamics, de Gruyter Exp. Math. 49, de Gruyter, Berlin, 2009.

[3] V. Anashin, A. Khrennikov and E. Yurova, Characterization of ergodicity of p-adic dynamical systems by using the van der Put basis, Dokl. Mat. 83 (2011), 306-308 (in Russian).

[4] J. Bryk and C. E. Silva, Measurable dynamics of simple p-adic polynomials, Amer. Math. Monthly 112 (2005), 212-232.

[5] Z. Coelho and W. Parry, Ergodicity of p-adic multiplications and the distribution of Fibonacci numbers, in: Topology, Ergodic Theory, Real Algebraic Geometry, Amer. Math. Soc. Transl. (2) 202, Amer. Math. Soc., 2001, 51-70.

[6] H. Diao and C. E. Silva, Digraph representations of rational functions over the p-adic numbers, $p$-Adic Numbers Ultrametric Anal. Appl. 3 (2011), 23-38.

[7] M. Gundlach, A. Khrennikov and K.-O. Lindahl, On ergodic behavior of p-adic dynamical systems, Infin. Dimens. Anal. Quantum Probab. Related Topics 4 (2001), 569-577.

[8] S. Jeong, Toward the ergodicity of p-adic 1-Lipschitz functions represented by the van der Put series, J. Number Theory 133 (2013), 2874-2891.

[9] E. Yurova, Van der Put basis and p-adic dynamics, p-Adic Numbers Ultrametric Anal. Appl. 2 (2010), 175-178.

\author{
Nacima Memić \\ Department of Mathematics \\ Faculty of Natural Sciences and Mathematics \\ University of Sarajevo \\ Zmaja od Bosne 33-35 \\ Sarajevo, Bosnia and Herzegovina \\ E-mail: nacima.o@gmail.com
}


